\section{Impact of emotion on memory}

\author{
Controlled study of the influence of emotionally charged \\ material on declarative memory in Alzheimer's disease
}

\author{
HIROAKI KAZUI, ETSURO MORI, MAMORU HASHIMOTO, \\ NOBUTSUGU HIRONO, TORU IMAMURA, SATOSHI TANIMUKAI, \\ TOKIJI HANIHARA and LARRY CAHILL
}

\begin{abstract}
Background In an earlier study we showed that a powerful emotional experience (the Kobe earthquake) reinforced memory retention in patients with Alzheimer's disease, but we could not control factors other than the emotional impact of the earthquake.
\end{abstract}

Aims To test our previous findings in a controlled experimental study.

Method Recall tests consisting of two short stories were administered to 34 patients with Alzheimer's disease and 10 normal subjects. The two stories were identical except for one passage in each story: one was emotionally charged (arousing story) and the other (neutral story) was not.

Results In both groups, the emotionally charged passage in the arousing story was remembered better than the counterpart in the neutral story. In addition, the extent of the memory improvement was similar in the subjects and in the controls.

Conclusions The results provide further evidence that emotional arousal enhances declarative memory in patients with Alzheimer's disease, and give a clue to the management of people with dementia.

Declaration of interest None.
It has been demonstrated that emotional arousal enhances declarative memory in healthy individuals and some amnesic patients (Bradley et al, 1992; Cahill \& McGaugh, 1995; Hamann et al, 1997a,b). Although memory impairment is a central and prominent feature of Alzheimer's disease, the effect of emotion on memory in this illness has not been well studied. We previously found that most of the patients with Alzheimer's disease who encountered a terrible earthquake remembered what happened around the time of the earthquake better than a less emotional event, a magnetic resonance imaging (MRI) study that they underwent, which suggests that fear reinforces memory retention in $\mathrm{Alz}$ heimer's disease (Ikeda et al, 1998). However, because the study design involved a naturalistic approach, it was difficult or impossible to control for some factors of the target events, other than the emotional impact of the event, which could have in fluenced memory retention. To re-evaluate our previous finding of an enhancement of declarative ${ }^{1}$ memory for emotional material in Alzheimer's disease, we conducted the present strictly controlled experimental study.

\section{METHOD}

\section{Subjects}

Full ethical approval was obtained for the project. A detailed description of the study was given to the subjects and relatives and written consent was obtained.

The subjects were 34 Japanese patients (27 women and 7 men) with

\footnotetext{
I. Long-term memory is differentiated into declarative memory and procedural memory. Declarative memory is explicit and conscious and it consists of information based on specific facts or data. Procedural memory is assessed by performance of particular skills and is usually retained in amnesia.
}

probable Alzheimer's disease and 10 normal elderly control subjects matched for age, gender and educational attainment. The normal controls were seven women and three men recruited from the community, who had no neurological signs or significant medical antecedents. The Mini-Mental State Examination (MMSE; Folstein et al, 1975) was administered to all normal subjects. The patients with Alzheimer's disease were recruited from those who were admitted to our hospital for examination, according to the criteria below. All patients were examined by both neurologists and psychiatrists during a short-term hospital stay and given routine laboratory tests and standard neuropsychological examinations; in addition, an electroencephalogram, an MRI brain scan, and MR angiograms of the neck and head were obtained. The inclusion criteria were those of the National Institute of Neurological and Communicative Disorders and Stroke/Alzheimer's Disease and Related Disorders Association for probable Alzheimer's disease (McKhann et al, 1984). The exclusion criteria were: (1) any complication due to other neurological or physical illness; (2) any MRI evidence of focal brain lesions and of cerebral arterial occlusive lesions on MR angiography; and (3) severe cognitive, attentional, or behavioural disorders which would make memory tests difficult. The severities of dementia as rated by the Clinical Dementia Rating (CDR; Hughes et al, 1982) were 0.5 (minimal) for four patients, 1 (mild) for 25, 2 (moderate) for four, and 3 (severe) for the remaining one. The MMSE and Alzheimer's Disease Assessment Scale - Cognitive sub-scale (ADAS cog , Mohs et al, 1983) were administered to all Alzheimer's subjects. Table 1 summarises the demographic and cognitive characteristics of the subjects.

The 34 Alzheimer's disease patients were divided into two groups: one group was read an arousing story, then, 2 weeks later, a neutral story ( $\mathrm{AD}-\mathrm{AN})$; the other was read a neutral story, followed 2 weeks later by an arousing story (AD-NA). The groups were comparable in age and gender, educational attainments, MMSE scores, and $\mathrm{ADAS}_{\mathrm{cog}}$ scores. The 10 normal elderly subjects (NC) were also divided into two similarly comparable groups (NC-AN and NC-NA) (except for ADAS $_{\text {cog }}$ scores). Table 2 summarises the demographic and cognitive characteristics of subjects in the four groups. 
Table I Demographic and cognitive characteristics of patients with Alzheimer's disease and normal controls

\begin{tabular}{lrr}
\hline & Patients & Controls \\
\hline Gender (male:female) & $7: 27$ & $3: 7$ \\
Age (mean (s.d.) years) & $71.6(6.5)$ & $70.8(5.5)$ \\
Education (mean (s.d.) years) & $9.4(2.6)$ & $9.2(2.1)$ \\
Mini-Mental State Examination score (mean (s.d.)) & $22.6(2.9)$ & $28.6(I .7)$ \\
Alzheimer's Disease Assessment Scale (Cognitive) score (mean (s.d.)) & $15.7(5.3)$ & NA \\
\hline
\end{tabular}

\section{ASSESSMENT}

We used the illustrated story paradigm that has been validated in many studies as an appropriate test for the assessment of emotionally influenced memory (Heuer \& Reisberg, 1990; Cahill et al, 1994; Cahill \& McGaugh, 1995; Adolph et al, 1997; Hamann et al, 1997a). In this paradigm, subjects viewed 11 colour photographs which were shown in sequence, accompanied by either an emotionally charged story narrative, or a narrative which was not emotionally charged. The emotionally charged story (arousing story) and the emotionally uncharged story (neutral story) were translated from the English of the original version into Japanese. Together, the photographs and narrative (one sentence per photograph) tell a story. The photographs were presented on a 17-inch Apple high-resolution colour monitor at a rate of 1 photograph every 20 seconds, controlled with the Aldus Persuasion version 3.0J (Adobe Inc., Mountain View, CA, USA) on the Power Macintosh 8100/ 80 (Apple Computer Inc., Cupertino, CA). A researcher (H.K.) next to the subjects read the stories while the photographs were shown.

Both types of story consisted of three phases. For the first four of the 11 photographs (phase 1), the narrations in the arousing story were identical to those in the neutral story. In this phase, both stories de- picted a mother taking her son to visit his father at work. In the middle four photographs (phase 2), the narrations in the arousing story differed from those in the neutral story: phase 2 of the arousing story was emotionally charged and phase 2 of the neutral story was not. In phase 2 of the arousing story, the boy was badly hurt in an automobile accident, and surgeons struggled to save his life; while in phase 2 of the neutral story, the boy watched a disaster drill in the hospital. For the last three photographs (phase 3), the narrations in the arousing story were nearly identical to those in the neutral story: phase 3 of both stories depicted the mother leaving the hospital. The arousing and neutral stories are closely matched in content, complexity and style, and were confirmed to be matched in comprehensibility (Cahill \& McGaugh, 1995).

The subjects were told to pay close attention to the photographs and the narrative and to remember the story. Immediately following the presentation of the story, they were asked to rate the emotional charge of the stories on a scale of 1-4 (1 indicating 'not emotional' and 4 indicating 'highly emotional').

After rating the emotional charge of the stories, and 5 minutes after the end of the presentation of the photographs, the subjects were given an 11-item recall test. We did not use the standard methods of the recall test, because the Alzheimer's disease patients' amnesia was severe. Instead, we used a simplified version of the recall paradigm. As the photographs were presented one by one again in the same order, subjects were asked questions about the story line (one question per photograph). If they could answer a question perfectly, they scored 2 points; if they could not answer at all, they scored 0 points; if their answer was partially correct, they scored 1 point. Thus, the maximum scores of phases 1,2 and 3 were 8,8 and 6 points. Recall scores were expressed as a percentage of the maximum of each phase, because of the different numbers of questions given for each phase.

The second part of the experiment was carried out 2 weeks later. On this occasion, those groups who had been read the arousing story 2 weeks previously were now read the neutral story, and those who had been read the neutral story 2 weeks previously were now read the arousing story. The scores in the AD-AN and AD-NA groups were evaluated together as scores of the Alzheimer's disease subjects, and the scores in the NC-AN and NC-NA groups were evaluated together as scores of the normal controls.

\section{Statistical analysis}

The rating of the emotional charge of the stories was analysed by using two-way analysis of variance (ANOVA) for repeated measures with one between-factor (group) and one within-factor (story). The recall scores across the three phases on the assessment of emotionally influenced memory was analysed by using three-way ANOVA for repeated measures, with one between-factor (group) and two within-factors (story and phase). To specifically elucidate the effect of emotional arousal on memory we carried out two-way ANOVA for repeated measures with two within-factor (story and phase) and a post hoc Tukey honestly significant

Table 2 Demographic and cognitive characteristics of subjects in four groups

\begin{tabular}{|c|c|c|c|c|}
\hline & AD-AN & AD-NA & NC-AN & NC-NA \\
\hline Gender (male:female) & $4: 13$ & $3: 14$ & $2: 3$ & $\mathrm{I}: 4$ \\
\hline Age (mean (s.d.) years) & $71.7(5.9)$ & $71.4(6.9)$ & $71.0(3.8)$ & $70.6(7.3)$ \\
\hline Education (mean (s.d.) years) & $9.5(3.2)$ & $9.4(1.8)$ & $9.2(1.8)$ & $9.2(2.7)$ \\
\hline Mini-Mental State Examination score (mean (s.d.)) & $22.6(2.8)$ & $22.5(2.7)$ & $28.4(1.1)$ & $28.8(2.2)$ \\
\hline Alzheimer's Disease Assessment Scale (Cognitive) score (mean (s.d.)) & $15.9(4.5)$ & $15.6(5.9)$ & NA & NA \\
\hline
\end{tabular}

AD-AN: Alzheimer's disease patients who were first read the arousing story and then the neutral story. AD-NA: Alzheimer's disease patients who were first read the neutral story and then the arousing story. NC-AN: normal controls who were first read the arousing story and then the neutral story. NC-NA: normal controls who were first read the neutral story and then the arousing story. 


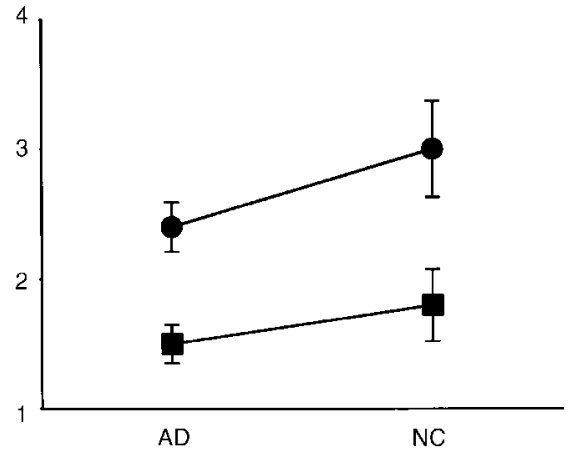

Fig. I Rating of emotional charge of the stories: the arousing story $(O)$ and the neutral story $(\square)$ for Alzheimer's disease (AD) and normal controls (NC). Error bars represent standard error of the mean

difference test. The statistically significant level was set at $P<0.05$.

\section{RESULTS}

\section{Ratings of the emotional charge of the stories}

The ratings of the emotional charge of the stories are illustrated in Fig. 1. The mean (s.d.) rating of the emotional charge of the arousing story for the Alzheimer's disease patients was 2.4 (0.9); that of the neutral story for the Alzheimer's patients 1.5 (1.1); that of the arousing story for the normal controls 3.0 (0.9); and of the neutral story for the normal controls 1.8 (1.2). The two-way ANOVA revealed a significant story effect $(F(1,42)=21.4, P<0.001)$. But group effect $(F(1,42)=2.60, P=0.11)$ and group $\times$ story interaction $(F(1,42)=0.61, P=0.44)$ were not significant. Thus, both the Alzheimer's patients and the normal controls rated the arousing story more emotional than the neutral story.

\section{Emotionally influenced memory test}

The results of the recall memory test for the three phases of the arousing and the neutral stories for the Alzheimer's patients and the normal controls are illustrated in Fig. 2. The three-way ANOVA revealed a significant group effect $(F(1,42)=94.0$, $P<0.0001)$, a significant story effect $(F(1,42)=12.3, \quad P<0.005)$, a significant phase effect $(F(2,84)=6.88, \quad P<0.005)$, and a significant story $\times$ phase interaction $(F(2,84)=30.1, P<0.001)$. But the group $\times$ story interaction $(F(1,42)=4.02, P=0.052)$, group $\times$ phase interaction $(F(2,84)=30.1$, $P=0.21$, and group $\times$ story $\times$ phase inter-

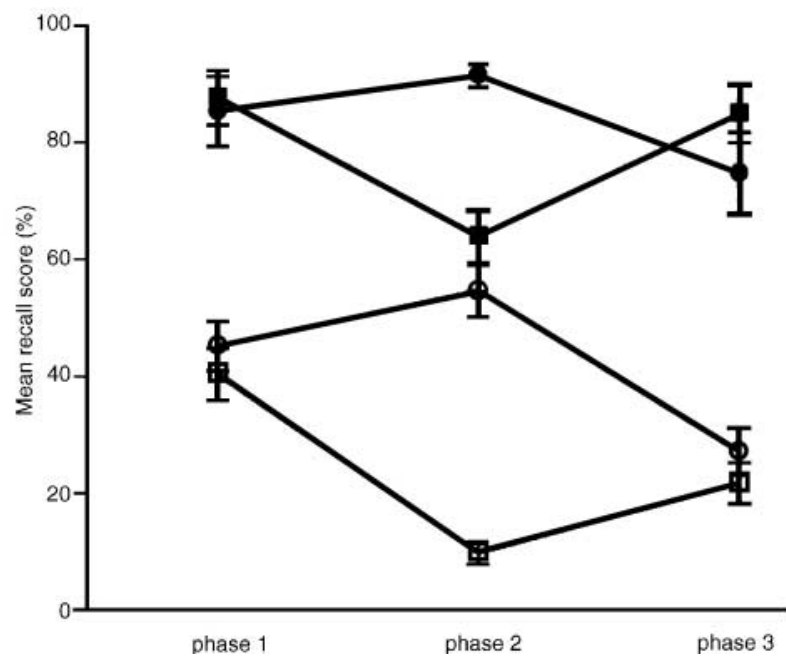

Fig. 2 Results of the recall memory test for the three phases of the arousing and neutral stories in patients with Alzheimer's disease and normal controls. $\bigcirc$, Alzheimer's patients read the arousing story; $\square$, Alzheimer's patients read the neutral story;, normal controls read the arousing story; $\square$, normal controls read the neutral story. Error bars represent standard error of the mean.

action $(F(2,84)=0.48, P=0.62)$ were not significant.

The two-way ANOVA for the Alzheimer's group revealed a significant story effect $\quad(F((1,34)=24.9, \quad P<0.001), \quad$ a significant phase effect $(F(2,68)=14.8$, $P<0.001$ ), and a significant story $\times$ phase interaction $(F(2,68)=37.5, P<0.001)$. The post hoc test showed that the recall scores in phase 2 were significantly worse for the neutral story than for the arousing story $(P<0.001)$, while the recall scores in phases 1 and 3 of the two stories were comparable. The analysis for the NC group revealed a significant story $\times$ phase interaction $(F(2,18)=12.0, P<0.001)$, but no significant story effect $(F(1,9)=3.25$, $P=0.10)$ or phase effect $(F(2,18)=1.8$, $P=0.25)$. The post hoc analysis showed that the recall scores in phase 2 were significantly worse for the neutral story than for the arousing story $(P<0.005)$, while the recall scores in phases 1 and 3 for the two stories were comparable.

\section{DISCUSSION}

\section{A paradigm for emotionally influenced memory}

Although both naturalistic and experimental approaches have merits, experimental studies have obvious advantages in the control of experimental variables and the assessment of the accuracy of memory. In our strictly controlled experimental study, we could control factors other than emotional impact which influenced memory retention, such as the content, complexity and comprehensibility of the target events, and the style and time of exposure to them. In addition, because the current paradigm was a more natural one, involving a connected series of pictured events, rather than a randomly ordered series of photographs, potential artefacts due to presenting a random selection of pictures were eliminated. Therefore, not only was the current paradigm a strictly controlled one, but it was able to assess the enhancing effect of emotional arousal on declarative memory in a condition close to daily life.

\section{Emotionally influenced memory in Alzheimer's disease}

In the present study, the rating of the emotional charge of the arousing story was significantly higher than that of the neutral story, in both the Alzheimer's patients and the normal subjects, reconfirming that both groups were similarly more emotionally charged by the arousing story than by the neutral story. The Alzheimer's disease patients recalled less about the stories than did the normal subjects, for both stories. A phase-by-phase comparison of the results in each group showed that the arousing story was better recalled than the neutral story only in phase 2 , in which the emotional impact in the arousing story was greater than that in the neutral one. These results strongly indicate that the enhancing effect of emotional arousal on 
declarative memory is preserved in patients with Alzheimer's disease, although the memory function itself in such patients was poorer than in normal subjects.

\section{Magnitude of enhancement}

From a neuroanatomical viewpoint, the amygdala is considered to play a crucial role in emotionally influenced memory (Cahill et al, 1996, 1998; Hamann et al, 1999). Adolph et al (1997) demonstrated the failure of the enhancing effect of emotional arousal on memory in patients with bilateral damage to the amygdala, using the same emotional memory paradigm that was used in the current study. In our previous study we reported that emotional remote memory about a terrible earthquake was significantly more closely correlated with the volume of the amygdala than with the volume of the hippocampus in Alzheimer's patients, indicating that the impairment of the enhancing effect of emotional arousal on memory in these patients is related to the degree of damage to the amygdala (Mori et al, 1999). That study also indicated that in some Alzheimer's patients, arousal material failed to enhance memory. However, in the present study, the magnitude of the enhancement of memory in Alzheimer's patients by emotional material was as good as that in normal subjects.

One possible reason for this discrepancy might be the difference in the severity of dementia in the target subjects. In the patients in the present study, dementias were milder than those in our previous study, as rated by the CDR. The volume of the amygdala decreased and the neuropathological changes in that region increased with increasing CDR score (Jack et al, 1997; Haroutunian et al, 1998). Because more of the amygdala was probably preserved in the patients in this study than in those in our previous study, those in the present study might have had a greater memory for emotional material.

Another possible reason for this discrepancy might be the easiness of the current recall test. The recall test in the present paradigm was made easier to enable us to assess the memory function fairly and to avoid the floor effect in Alzheimer's disease. Consequently, the recall test might have become too easy for the normal subjects, and a ceiling effect might have occurred in phase 2

\section{CLINICAL IMPLICATIONS}

- Patients with Alzheimer's disease are influenced by an emotionally charged story.

- The enhancing effect of emotional arousal on declarative memory is preserved in patients with Alzheimer's disease.

- The findings have implications for the management of people with dementia: for example, for care, rehabilitation and drug intervention to enhance memory.

\section{LIMITATIONS}

There was no neuropathological confirmation of the clinical diagnosis of Alzheimer's disease.

Because the patients included in this study mostly had mild dementia, the enhancing effect of emotional arousal on declarative memory in patients with moderate to severe Alzheimer's disease was not established.

- The long-term enhancing effect of emotional arousal on declarative memory in patients with Alzheimer's disease was not examined.

HIROAKI KAZUI, MD, ETSURO MORI, MD, MAMORU HASHIMOTO, MD, NOBUTSUGU HIRONO, MD, TORU IMAMURA, MD, SATOSHI TANIMUKAI, MD, TOKIJI HANIHARA, MD, Department of Clinical Neurosciences, Hyogo Institute for Aging Brain and Cognitive Disorders, Himeji, Japan; LARRY CAHILL, PhD, Department of Neurobiology and Behavior and Center for the Neurobiology of Learning and Memory, University of California, Irvine, USA

Correspondence: Dr Hiroaki Kazui, Department of Clinical Neurosciences, Hyogo Institute for Aging Brain and Cognitive Disorders, 520 Saisho-ko, Himeji, 670-098I, Japan. Tel.: +8I 79295 55II; fax: +8I 79295 8199; e-mail: kazui@hiabcd.go.jp

(First received 23 November 1999, final revision 27 March 2000, accepted 4 April 2000)

for the arousing story in the normal subjects, which would have artificially decreased the degree to which the arousing story enhanced their memory.

\section{Clinical implications of emotionally influenced memory}

The finding that emotional arousal enhanced memory in Alzheimer's disease has implications for the management of people with dementia. It directly suggests that they should be treated gently, to avoid eliciting strong negative feelings. Although Alzheimer's patients forget less emotional events, they have a better memory for emotionally negative events, and this might damage the caregiver-patient relationship, for example. Although the emotional arousal used in the present study was negative in nature, both positive and negative emotional arousal have similar memory-enhancing effects (Hamann et al, 1997b). In consideration of our finding, together with this evidence of Hamann et al, it is suggested that the instructions and guidance given to Alzheimer's disease to patients may be easier to remember if emotionally arousing actions, devices and so on are used.

The present finding also implies a potentially useful strategy for memory rehabilitation. Furthermore, the fact that emotional arousal enhances memory raises the possibility of enhancing memory without emotional arousal, by using agents such as the $\beta$-adrenergic receptor agonist and the corticosterone receptor agonist, which involve the final emotion-memory pathway (Cahill et al, 1998). Clearly, more research is needed to determine the association between emotion and memory and to develop a useful strategy for memory enhancement. 


\section{REFERENCES}

Adolph, R., Cahill, L., Schul, R., et al (1997) Impaired declarative memory for emotional material following bilateral amygdala damage in humans. Learning and Memory, 4, 291-300.

Bradley, M. M., Greenwald, M. K., Petry, M. C., et al (1992) Remembering pictures: pleasure and arousal in memory. Journal of Experimental Psychology: Learning, Memory, and Cognition, 18, 379-390.

Cahill, L. \& McGaugh, J. L. (1995) A novel demonstration of enhanced memory associated with emotional arousal. Consciousness and Cognition, $\mathbf{4}$. 410-421.

— \& _ (1998) Mechanism of emotional arousal and lasting declarative memory. Trends in Neurosciences, $\mathbf{2 l}$, 294-299.

— , Prins, B., Weber, M., et al (1994) $\beta$-Adrenergic activation and memory for emotional events. Nature, 37I, 702-704

_, Haier, R. J., Fallon, J., et al (1996) Amygdala activity at encoding correlated with long-term, free recall of emotional information. Proceedings of the
National Academy of Sciences of the United States of America, 93, 8016-8021.

Folstein, M. F., Folstein, S. E. \& McHugh, P. R. (1975) 'Mini-Mental State'. A practical method for grading the cognitive state of patients for the clinician. Journal of Psychiatric Research, 12, 189-198.

Hamann, S. B., Cahill, L., McGaugh, J., et al (1997a) Intact enhancement of declarative memory for emotional material in amnesia. Learning and Memory, 4 , 301-309.

_, _ \& Squire, L. R. (1997b) Emotional perception and memory in amnesia. Neuropsychology, II, 104-113.

_ , Ely, T. D., Grafton, S. T., et al (1999) Amygdala activity related to enhanced memory for pleasant and aversive stimuli. Nature Neuroscience, 2, 289-293.

Haroutunian, V., Perl, D. P., Purohit, D. P., et al (1998) Regional distribution of neuritic plaques in the nondemented elderly and subjects with very mild Alzheimer disease. Archives of Neurology, 55, ||85-|19|.

Heuer, F. \& Reisberg, D. (1990) Vivid memories of emotional events: the accuracy of remembered minutiae. Memory \& Cognition, 18, 496-506.
Hughes, C. P., Berg, L., Danziger, W. L., et al (1982) A new clinical scale for the staging of dementia. British Journal of Psychiatry, 140, 566-572.

Ikeda, M., Mori, E., Hirono, N., et al (1998) Amnestic people with Alzheimer's disease who remembered the Kobe earthquake. British Journal of Psychiatry, 172 425-428.

Jack, C. R., Jr., Petersen, R. C., Xu, Y. C., et al (1997) Medial temporal atrophy on MRI in normal aging and very mild Alzheimer's disease. Neurology, 49, 786-794.

McKhann, G., Drachman, D., Folstein, M., et al (1984) Clinical diagnosis of Alzheimer's disease: report of the NINCDS-ADRDA Work Group under the auspices of Department of Health and Human Services Task Force on Alzheimer's Disease. Neurology, 34, 939-944.

Mohs, R. C., Rosen, W. G. \& Davis, K. L. (1983) The Alzheimer's Disease Assessment Scale; an instrument for assessing treatment efficacy. Psychopharmacology Bulletin, 19, 448-450.

Mori, E., Ikeda, M., Hirono, N., et al (1999) Amygdalar volume and emotional memory in Alzheimer's disease. American Journal of Psychiatry, 156 216-222. 